DRAFT VERSION JUNE 30, 2021

Preprint typeset using LTEX style emulateapj v. 04/20/08

\title{
THE IMPACT OF INHOMOGENEOUS REIONIZATION ON THE SATELLITE GALAXY POPULATION OF THE MILKY WAY
}

\author{
Michael T. Busha, Marcelo A. Alvarez, Risa H. Wechsler, Tom Abel, Louis E. Strigari \\ Kavli Institute for Particle Astrophysics and Cosmology \\ Stanford University, Stanford, CA, 94305 \\ SLAC National Accelerator Laboratory, Menlo Park, CA, 94025 \\ mbusha, malvarez, rwechsler, tabel, strigari@stanford.edu \\ Draft version June 30, 2021
}

\begin{abstract}
We use the publicly available subhalo catalogs from the via Lactea II simulation along with a Gpc-scale N-body simulation to understand the impact of inhomogeneous reionization on the satellite galaxy population of the Milky Way. The large-volume simulation is combined with a model for reionization that allows us to predict the distribution of reionization times for Milky Way mass halos. Motivated by this distribution, we identify candidate satellite galaxies in the simulation by requiring that any subhalo must grow above a specified mass threshold before it is reionized; after this time the photoionizing background will suppress both the formation of stars and the accretion of gas. We show that varying the reionization time over the range expected for Milky Way mass halos can change the number of satellite galaxies by roughly two orders of magnitude. This conclusion is in contradiction with a number of studies in the literature, and we conclude that this is a result of inconsistent application of the results of Gnedin (2000); subtle changes in the assumptions about how reionization affects star formation in small galaxies can lead to large changes in the effect of changing the reionization time on the number of satellites. We compare our satellite galaxies to observations using both abundance matching and stellar population synthesis methods to assign luminosities to our subhalos and account for observational completeness effects. Additionally, if we assume that the mass threshold is set by the virial temperature $T_{\text {vir }}=8 \times 10^{3} \mathrm{~K}$ we find that our model accurately matches the $v_{\max }$ distribution, radial distribution, and luminosity function of observed Milky Way satellites for a reionization time $z_{\text {reion }}=8_{-2}^{+3}$, assuming that the via Lactea II subhalo distribution is representative of the Milky Way. This results in the presence of $540_{-340}^{+100}$ satellite galaxies.

Subject headings: cosmology:theory — large-scale structure of universe — dark matter
\end{abstract}

\section{INTRODUCTION}

While the cold dark matter (CDM) paradigm has been very successful in explaining the large scale distribution of matter in the universe, one final test lies in its ability to predict the distribution of matter on small scales, including the distribution of satellite galaxies around the Milky Way. In the hierarchical model of structure formation, massive objects such as the Milky Way halo are built up through a series of mergers where small, dense objects collapse early and merge to form larger objects. High-resolution numerical simulations have shown that the dense cores from a significant number of these small building blocks should survive today as gravitationally bound subhalos (Klypin et al. 1999; Moore et al. 1999; Diemand et al.|2008; Springel et al.|2008). From these initial results (Klypin et al. 1999; Moore et al.1999) and from semi-analytic modeling (Kauffmann et al. 1993) it was also clear that there are far fewer luminous dwarf satellites around the Milky Way than bound dark matter subhalos in the simulations.

There has been no shortage of solutions proposed to explain this discrepancy, including those which modify the dark matter power spectrum to reduce the amount of small scale power through warm dark matter-like models (e.g., Colín et al. 2000; Bode et al. 2001; Zentner \& Bullock 2003; Busha et al. 2007). Less exotic models, however, such as the presence of a number of "dark-dark halos," dark matter subhalos that do not host galaxies, provide a well-motivated resolution to this issue within the CDM paradigm. In particular, the presence of photoionizing radiation is expected to have a significant effect on the ability of a subhalo to host a luminous galaxy (Bullock et al. 2000; Gnedin 2000; Benson et al. 2002; Somerville 2002; Dekel \& Woo 2003; Shaviv \& Dekel 2003; Li et al. 2008; Madau et al. 2008; Koposov et al. 2009). Star formation in dark matter halos in the early universe is only expected to be able to begin once the halo has grown massive enough to cool efficiently by atomic cooling, typically around $T_{\text {vir }} \sim 10^{4} \mathrm{~K}$. However, before many halos are able to reach this mass, the universe enters the phase of reionization, in which photoionizing UV radiation is released by the early generations of stars and quasars. This ionizing radiation heats the halo gas to a temperature of a few times $10^{4} \mathrm{~K}$, preventing it from being pulled into the shallow potential wells of halos with virial temperatures lower than $\sim 10^{5} K$ (Thoul \& Weinberg 1996; Diikstra et al. 2004), effectively suppressing further star formation.

Recent analysis of the Sloan Digital Sky Survey (SDSS) has resulted in the discovery of a large number of lowsurface brightness dwarf galaxies (Willman et al. 2005a b; Belokurov et al. 2006; Zucker et al. 2006a b; Belokurov et al. 2007; Irwin et al. 2007; Walsh et al. 2007; Belokurov et al. 2008), which is now beginning to shed a new light on the mapping of galaxies onto dark matter halos at the lowluminosity end. Several of these newly-discovered satellites have luminosities similar to those of the least luminous globular clusters, and a dynamical analysis indicates that they have the largest mass-to-light ratio of any known galaxies (Martin et al. 2007; Simon \& Geha 2007; Strigari et al. 2008; Geha et al. 2008). Given both the magnitude limit and 
sky-coverage fraction of the SDSS survey, it is certainly reasonable to assume that we have only detected a fraction of the Milky Way satellites (Koposov et al. 2008; Tollerud et al. 2008; Walsh et al. 2009), and there are exiting prospects for discovery of more satellites in future deep and wide surveys Abbott et al. 2005; Keller et al. 2007; Ivezic et al. 2008). A full understanding of the mapping between luminous satellite galaxies and dark matter subhalos will require a measurement of the luminosity distribution, radial distribution (Kravtsov et al. 2004b; Willman et al. 2004), and the kinematic properties of the satellites (Strigari et al. 2007b).

Recently, it has become understood that the universe actually reionizes quite inhomogeneously, even on very large scales (100 Mpc-1 Gpc; e.g., Sokasian et al. 2003; Barkana \& Loeb 2004; Iliev et al. 2006; Lidz et al. 2007; Alvarez et al. 2008). Given constraints on the global reionization history, e.g. from the cosmic microwave background polarization (e.g., Spergel et al. 2006), there is still a significant uncertainty in the precise reionization epoch of the matter in the Milky Way. In particular, the calculations of Alvarez et al. (2008) indicate that there is substantial scatter in the reionization histories of halos of a given mass, and that on average Milky Way mass halos reionization redshifts have approximately the same distribution as that of the universe as a whole. A change in the reionization time of the Milky Way may have a dramatic impact on its satellite population because the reionization history may directly affect the ability of a subhalo to reach the size where gas is able to cool and begin the star formation process. The primary aim of this paper is to understand what, if any, effect the reionization epoch of a given Milky Way halo has on its satellite population.

The effect of the reionization epoch on the satellite population has been previously addressed in the literature, with most studies finding little effect (e.g., Somerville 2002; Kravtsov et al. 2004b). These studies primarily have addressed the luminosity range of the "classical dwarfs" in the Milky Way. Given the dramatically different observational picture that has emerged with new observations from SDSS, combined with the possible importance of a spread in reionization epochs expected from inhomogeneous reionization, we re-investigate this question here. We combine a highresolution dark matter simulation (the via Lactea II simulation of Diemand et al. 2007b, 2008) with various assumptions about star formation in small halos, and compare to up-to-date constraints from the full observed satellite galaxy population. In this work, we critically examine the assumptions about the rate at which photoionizing UV radiation is able to heat halo gas. We find that the exact time a halo reionizes can have a significant impact on the satellite population, and use comparisons with the Milky Way's satellite distribution to constrain the reionization time of our own halo.

In section $\S 2$ we discuss our simulation and models, including methods for identifying subhalos that host satellite galaxies, and determining magnitudes of these galaxies. In $\S 3$ we discuss the observational sample of satellite galaxies that we compare to our model, including measurements and corrections for the luminosity, $v_{\max }$ and radial distribution functions. In $\S 4$ we directly compare our modeled and observed samples in a manner that fairly accounts for the incompleteness of the observations. In $\$ 5$ we discuss how our results compare with previous work in the literature, in particular addressing the differences between our results and those that have found little change in the satellite population for varying reionization times (Somerville 2002; Kravtsov et al. 2004b). Finally, we present our conclusions in $\S 6$.

\section{SIMULATIONS AND MODELING}

\subsection{Modeling the Large-scale Structure of Reionization}

In order to understand the distribution of reionization times of Milky Way sized galaxies, including any effect this may have on the satellite galaxy population, it is necessary to understand the distribution of dark matter on the largest scales. We use the recent results of Alvarez et al. (2008), in which the reionization process was modeled using an $\mathrm{N}$-body simulation of a $1 \mathrm{Gpc} h^{-1}$ box combined with an analytic prescription for predicting the reionization time for all points in the box, as described below. For more details on the reionization simulation and the halo correlation, see Alvarez et al. (2008).

The N-body simulation used the code Gadget2 (Springel 2005) to evolve $1120^{3}$ dark matter particles in a cosmology with $\Omega_{m}=0.25, \Omega_{\Lambda}=0.75$, and $\sigma_{8}=0.8$, with particle mass resolution $M_{p}=4.94 \times 10^{10} h^{-1} \mathrm{M}_{\odot}$. The initial conditions were generated using the 2 nd order Lagrangian perturbation code 2LPT (Crocce et al. 2006). A Friends-of-Friends group finder based on the Ntropy framework (Gardner et al. 2007) was run on the $z=0$ output and identified all halos with $M_{F o F} \geq 1.58 \times 10^{12} h^{-1} \mathrm{M}_{\odot}=32$ particles. This simulation was run in conjunction with the LasDamas collaboration ${ }^{1}$. The reionization history for this simulation was then calculated using the density field of the initial conditions. Working at a single point in space, the dark matter density field is smoothed over a series increasing radii and use the EPS formalism to ask, for a given redshift, what is the smallest (if any) radius at which the fraction of collapsed mass in halos greater than some threshold is greater than a specified fraction, $1 / \zeta_{c}$. The reionization time, $z_{\text {reion }}$, for that point is the earliest redshift at which this criteria is first met for any radius. For this simulation we use $\zeta_{c}=10$ and a threshold mass $10^{8} \mathrm{M}_{\odot}$. Combined with the group catalog, this results in a reionization time for each halo in the simulation.

\subsection{Modeling Galaxy Formation}

While the above simulations are sufficient for measuring the reionization history of Milky Way mass halos, significantly higher mass resolution is needed to understand the subhalo distribution. For this, we use the publicly available mass accretion histories ${ }^{2}$ from the high-resolution via Lactea II simulation (Diemand et al. 2007a b, 2008). This simulation models a single dark matter halo with virial mass $M_{\text {halo }}=1.9 \times 10^{12} h^{-1} \mathrm{M}_{\odot}$ that is able to resolve subhalos down to mass limit $M_{s u b}>10^{6} h^{-1} \mathrm{M}_{\odot}$. The publicly available data includes the distribution, tidal mass, and $v_{\max }$ histories for the most massive progenitors of all $z=0$ subhalos back to redshift $z=28$.

In order to connect the via Lactea II dark matter subhalo population to a satellite galaxy population, we assume that stars begin forming once atomic cooling becomes effective, when a halo shock heats to virial temperature $T_{\text {vir }} \approx$ $8 \times 10^{3} \mathrm{~K}$, but that reionization heats the gas in the subhalos to the point where this becomes ineffective (Thoul \& Weinberg 1996; Kepner et al. 1999; Wise \& Abel 2008). We treat this heating as an instantaneous process (see section 5 for a discussion), causing reionization to end star formation for the

\footnotetext{
${ }^{1}$ http://lss.phy.vanderbilt.edu/lasdamas/

2 http://www.ucolick.org/ diemand/vl/data.html
} 
vast majority of the $\sim 2500$ potential satellite galaxies; for these low-mass halos, all star formation must happen before $z_{\text {reion }}$. With this in mind, we can define a subhalo as being a satellite galaxy using a two parameter model: A subhalo must grow to a threshold mass, $M_{\mathrm{t}}$, above which HI cooling will allow star formation, before the host halo reionizes at $z_{\text {reion }}$ in order to host a satellite.

While we demonstrate the effects of varying both parameters in the next section, the work of Abel et al. (2002) uses high resolution AMR simulations to model the formation of the first stars and indicates that we anticipate $M_{\mathrm{t}} \approx 10^{6}-$ $10^{7} h^{-1} \mathbf{M}_{\odot}$. It is important to note that this process of hydrogen cooling simply defines a minimum mass of the population of the dark matter subhalos that could host satellite galaxies. However, this work predicts the stars forming in these halos to be very massive and short-lived. As such these very first star forming halos cannot be the direct progenitors of Milky Way satellites, which are observed to be metal-enriched objects with stars presumably of masses less than a solar mass. More relevant here are the calculations of Wise \& Abel (2008), who followed the build up of halos up to the masses when they start cooling via Lyman-alpha from neutral hydrogen. They included the radiative as well as the supernova feedback from the first generation of massive stars. The short-lived sources keep ionizing the baryonic material in the halos they form in, as well as their surroundings. However, as they turn off, material can cool again and repopulate the dark matter halos. So while the baryon fraction (Fig. 4 in Wise \& Abel 2008) fluctuates and decreases at times to as little as $10 \%$, star formation can continue as long as no sustained external UV flux sterilizes the halo. The latter case severely limits star formation and has been discussed many time in the literature (e.g., Babul \& Rees 1992; Thoul \& Weinberg 1996; Kepner et al. 1999; Dijkstra et al. 2004). It seems clear then from the limited guidance we have from numerical simulations that most Milky Way satellite halo progenitors experienced most of their star formation before they are permanently ionized.

Once we have identified satellite galaxies in the simulation, we must assign magnitudes to them in order to make direct comparisons with observations and to account for observational completeness effects. This is done using two methods. First, we use a halo abundance matching method Kravtsov et al. 2004a; Blanton et al. 2008). Here, luminosities are assigned to halos by assuming a one-to-one correspondence between $n\left(<M_{V}\right)$, the observed number density of galaxies brighter than $M_{v}$, with $n\left(>v_{\max }\right)$, the number density of simulated halos with maximum circular velocities larger than $v_{\max }$. For the distribution of magnitudes, we use the double-Schechter fit of Blanton et al. (2005) for low luminosity SDSS galaxies in the $g-$ and $r$-bands down to $M_{r}=-12.375$. The $v_{\max }$ values are taken from the halo catalog of a $160 \mathrm{Mpc} / \mathrm{h}$ simulation complete down to $v_{\max } \approx 90 \mathrm{~km} / \mathrm{s}$. In order to extrapolate this to lower circular velocities, we calculate a power-law fit to the low end of the $d n / d v_{\max }$ function. The resulting correspondence is shown in Figure 1 for the $r-, g-$, and $V$-bands (red, green, and black curves). The $V$ band magnitudes are calculated using the transformation $V=$ $g-0.55(g-r)-0.03$ from Smith et al. (2002). This method implicitly assumes that all galaxies have average color. Since the data from Blanton et al. (2005) is not deep enough to map onto the dwarf galaxy distribution, we use a power law to extrapolate the $M_{V}\left(v_{\max }\right)$ relation to lower magnitudes. For the

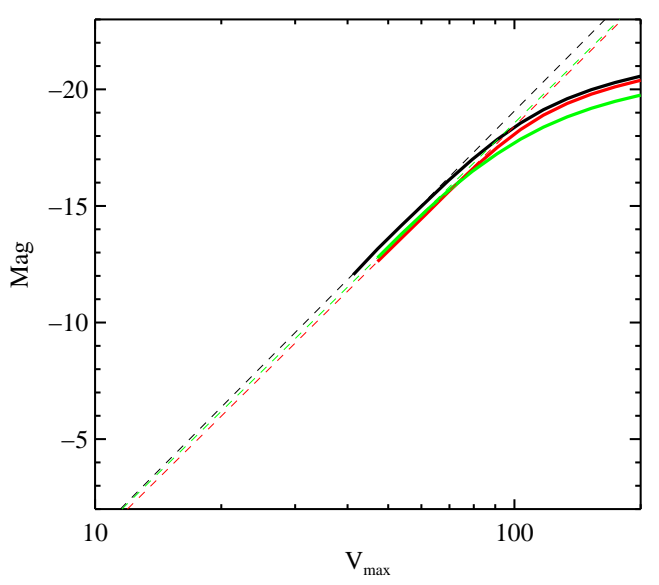

FIG. 1. - The relationship between magnitude and $v_{\max }$ for the $r-, g-$, and $V$ - bands using abundance matching (solid red, green and black lines). The dashed lines show power law fits to the low-luminosity end.

$V$-band, we get

$$
M_{V}-5 \log (h)=18.2-2.5 \log \left[\left(\frac{v_{\max }}{1 \mathrm{~km} / \mathrm{s}}\right)^{7.1}\right] .
$$

When selecting the appropriate $v_{\max }$ for assigning a luminosity, we follow the method of Conroy et al. (2006) and choose the peak $v_{\max }$ over the trajectory of the subhalo for subhalos that eventually cross the $10^{5} \mathrm{~K}$ post-reionization star forming threshold. For subhalos that never reach this threshold, we use the value of $v_{\max }$ at $z_{\text {reion. }}$. In both cases, this then corresponds roughly to the mass the halo had at the redshift they stopped rapidly forming stars.

The appeal of this method is that we are able to ignore much of the poorly understood (and poorly simulated) physics of galaxy formation using a statistical method that has been shown to, on average, reproduce a wide variety of observable properties for more massive galaxies Conroy et al.2006; Conroy \& Wechsler 2009), as well as some properties of dwarf galaxies down to $v_{\max } \sim 50 \mathrm{~km} / \mathrm{s}$ (Blanton et al. 2008). It is still unclear how this method will fare at lower masses; it must break down for small halos once they no longer host one galaxy on average. If this transition is sharp, however, it may be a reasonable approximation for most of the mass range where halos host galaxies.

As a second approach for assigning magnitudes, we use a toy model to predict the star formation rate and stellar mass of a satellite combined with the stellar population synthesis (SPS) code of Bruzual \& Charlot (2003) ${ }^{3}$. Here, we again assume that star formation begins when the satellite first crosses the mass threshold, $M_{\mathrm{t}}$, and ends at the reionization time, $z_{\text {reion. }}$. During this period, the star formation rate is set by the dark matter mass of the subhalo,

$$
S F R= \begin{cases}\epsilon\left(f_{\text {coldgas }} \frac{M_{D M}}{1 \mathrm{M}_{\odot}}\right)^{\alpha} & \text { if } M_{D M}>M_{\mathrm{t}}, z>z_{\text {reion }} \\ 0 & \text { otherwise }\end{cases}
$$

where $f_{\text {coldgas }}$ is the fraction of cold gas in the halo, and $\alpha$ and $\epsilon$ are free parameters. This is similar to model 1B of Koposov et al. (2009), with a couple of key differences. First, we impose a hard truncation of star formation at the epoch of reionization, something they only consider using

\footnotetext{
${ }^{3}$ http://www.cida.ve/ bruzual/bc2003
} 
their model where stellar mass is a constant fraction of dark matter mass. Second, they treat stellar mass as being proportional $M_{D M}^{\alpha}$ at some epoch, while we take the total stellar mass to be proportional to the integral of this quantity, as motivated by observations (e.g., Juneau et al. 2005; Noeske et al. 2007; Zheng et al. 2007; Conroy \& Wechsler 2009). For our model, we hold $f_{\text {coldgas }}$ constant and set it equal to the universal baryon fraction during the period of active star formation. Our two free parameters $\epsilon$ and $\alpha$, set the efficiency and scaling of the star formation. We keep $\epsilon$ as a free parameter that is used to match the luminosity function, but fix $\alpha=2$, extrapolated from higher mass galaxies at both low and high redshifts (Figure 8 of Conroy \& Wechsler 2009; Drory \& Alvarez 2008). The implicit assumption of this model is that subhalos contain a gas fraction equal to the universal baryon fraction, and that this gas exists in one of two phases. At early times the gas in low mass halos has a temperature less than $T=8 \times 10^{3} \mathrm{~K}$ and therefore cannot cool by atomic hydrogen cooling. However, once a halo reaches $M_{\mathrm{t}}$, cooling becomes effective and all gas rapidly enters a cold phase where it is able to form stars. Reionization, however, rapidly heats all gas in the subhalo to a few times $10^{4} \mathrm{~K}$, quenching star formation. The more massive halos eventually shock heat to such a virial temperature, which allows the ionized gas to cool again and resume star formation. Only 7 subhalos in the via Lactea II simulation ever reach such a mass. Regardless of mass, all star formation is ended once a satellite accretes onto the larger host halo, as we assume that this process causes all gas to be stripped from the subhalo. Most of the $z=0$ subhalos in via Lactea II were accreted in the range $z=0-6$. This model does ignore a large number of physical processes, such as recombinations and feedback, but much of this can likely be accounted for by appropriately setting the constants $\epsilon$ and $\alpha$. In Section $\$ 5$ we discuss how this model compares with previous studies. Satellite magnitudes for this model are determined using the stellar population synthesis code of Bruzual \& Charlot (2003). For this model, we treat the star formation as a series of bursts, all with the IMF of Chabrier (2003) and metallicity $Z=0.0004 Z_{\odot}$, broadly consistent with the expected level of enrichment from the earliest, most massive stars that preenrich the halo gas (Abel et al. 2002; Wise \& Abel 2008). Using these magnitudes, we tune the $\epsilon$ parameter of equation 2 so that our model most accurately reproduces the observed luminosity function of local satellite galaxies (see below). This results in typical values for $\epsilon \sim 10 \mathrm{M}_{\odot} / \mathrm{yr}$.

As a direct comparison, the resulting average $M_{V}\left(v_{\max }\right)$ relation from these two methods do not differ significantly. When considering the populations as a whole, the SPS method results in a weaker magnitude trend with $v_{\max }$ and slightly reduced magnitude range. On a galaxy by galaxy basis, however, this is appreciable scatter, with galaxy magnitudes differing by as much as 1.5 dex.

\section{OBSERVATIONAL SAMPLE}

In the next section we present a number of comparisons with the observed properties the Milky Way satellites. We take as our data set the 11 classical and 12 newly-identified SDSS dwarf galaxies within the fiducial milky way virial radius of $417 \mathrm{kpc}$ as presented in Table I of Tollerud et al. (2008). However, we exclude Boötes II from our comparisons of the maximum circular velocity, $v_{\max }$, because there are only poor constraints. When using the SDSS dwarfs, we must account for both the sky coverage of SDSS and the detection efficiency for each galaxy (Koposov et al. 2008). While it is

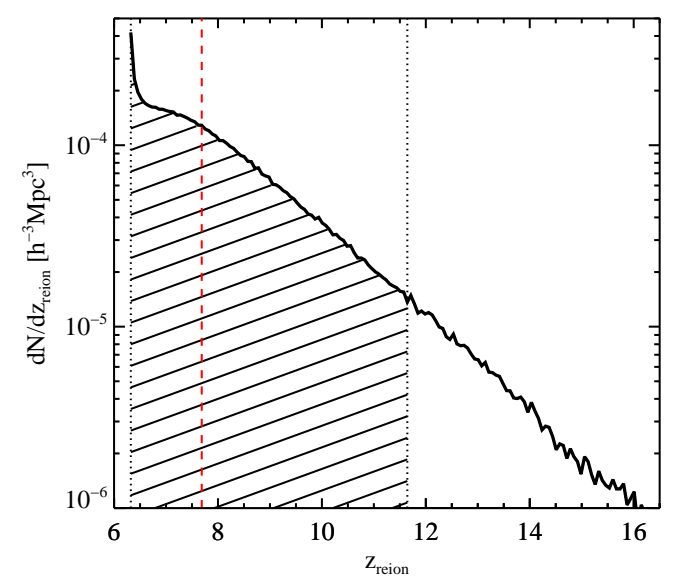

FIG. 2.- Distribution of reionization epochs for Milky Way mass halos, $M_{F o F}=1.6-2 \times 10^{12} h^{-1} \mathrm{M}_{\odot}$. The dotted lines and hatched region indicate the $95 \%$ limits for the distribution. See Alvarez et al. (2008) for more details on the simulations used to generate this distribution. The dashed red line indicates the median value $z_{\text {reion }}=7.7$.

straight-forward to adjust the observational data to account for sky coverage, a more subtle issue arises with the depth of SDSS. Because the survey is magnitude limited, SDSS is only complete in searching for satellite galaxies of absolute magnitude $M_{v}$ out to some radius $R_{\text {comp }}$. This completeness depth is roughly independent of surface brightness and the relation is given in Koposov et al. (2008) as

$$
R_{\text {comp }}\left(M_{v}\right)=\left(\frac{3}{4 \pi f_{D R 5}}\right)^{1 / 3} 10^{\left(-a M_{v}-b\right) / 3} \mathrm{Mpc}
$$

where $f_{D R 5}=0.194$ is the sky coverage fraction of SDSS, and $\mathrm{a}=0.6$ and $\mathrm{b}=5.23$. Tollerud et al. (2008) also used the via Lactea II simulation to calculate a correction due to this effect and use it to correct the luminosity function. However, this correction is unable to account for the radial or $v_{\max }$ distributions. Consequently, whenever we compare our model predictions with observations, we present the sky coveragecorrected distribution of observed satellites and a sub-sample of the simulated galaxies that is cut by the magnitude limit of equation 3 .

In addition to survey depth, the observational sample of Milky Way dwarfs is also limited by a surface brightness detectability constraint. This is discussed in detail in Koposov et al. (2008) who parameterize the detection efficiency of a satellite according to

$$
\epsilon\left(M_{v}, \mu\right)=G\left(\frac{M_{v}-M_{v, \text { lim }}}{\sigma_{M}}\right) G\left(\frac{\mu_{v}-\mu_{v, l i m}}{\sigma_{\mu}}\right),
$$

where $G(x)=0.5 \operatorname{erfc}(x / \sqrt{2})$ and $M_{v, \text { lim }}, \mu_{\text {lim }}, \sigma_{M}$, and $\sigma_{\mu}$ describe the magnitude and surface brightness limits of observations. Using their parameters for fitting current observations, we calculate the detection efficiency of our model galaxies using the conservative assumption that the light of a satellite is uniformly distributed out to the tidal radius of its host dark matter subhalo.

Finally, we must account for the observational uncertainties in measurements of the magnitudes of the dimmest dwarf galaxies. Effectively, the small number of stars used to determine the magnitude of a faint dwarf galaxy introduces shotnoise effects, creating additional uncertainty on the measurement. This is studied in detail in Martin et al. (2008), who 
report magnitude uncertainties in their Table 1 . We include these uncertainties when making comparisons between our modeled luminosity function and observations.

\section{RESULTS}

\subsection{Distribution of reionization epochs}

First, we first investigate the distribution of reionization times for Milky-Way size halos in Figure 2 The solid line of the Figure shows the distribution of reionization epochs for the $\sim 500,000$ identified halos with mass $M_{F o F}=1.6-2 \times$ $10^{12} h^{-1} \mathrm{M}_{\odot}$ from our Gpc simulation. From this plot, we see a wide distribution of reionization epochs, peaked around $z=6$ with a tail extending beyond $z=16$, fit by an exponential, $d N / d z_{\text {reion }} \propto e^{-0.6 z_{\text {reion }}}$. Ninety-five percent of the halos are reionized in the range $z_{\text {reion }}=6.3-11.6$ with a median redshift of 7.7. This distribution is also quite consistent with similar predictions from Weinmann et al. (2007). Percolation of reionization happens at $z=6.3$ and the universe rapidly becomes fully ionized, which is responsible for the sharp cutoff in the distribution. This broad distribution indicates that a precise constraint on the globally-averaged reionization epoch, for example as measured from the optical depth to the cosmic microwave background, does not give precise constraints on the reionization history of our local galaxy and its progenitors. As we will show, determining where the Milky Way itself sits in this distribution may be important for understanding its satellite population. The reionization epoch of a given halo is almost certainly correlated with other properties, including the large scale bias and the detailed formation history of the halo, and there may be observational clues beyond those presented here, to where the Milky Way lies in the distribution. We postpone an investigation of these issues to future work.

\subsection{Distribution of satellite populations}

Using the distribution of $z_{\text {reion }}$ from Figure 2 as a guide, we show in Figure 3 the number of subhalos hosting satellites galaxies, $n_{\text {sats, }}$, in the via Lactea II simulation for our model. The contours of the lower panel show the full variation in $n_{\text {sats }}$ as a function of both $z_{\text {reion }}$ and $M_{\mathrm{t}}$. The dashed lines denote constant virial temperatures. The thick lower line represents $T_{\text {vir }}=8 \times 10^{3} \mathrm{~K}$, the temperature where HI cooling is expected to be effective for gas that has not been photoionized (Wise \& Abel 2008). The upper line is $10^{5} \mathrm{~K}$, the mass where the halo has shock heated to the point where photoionized gas can cool (e.g., Haiman \& Bryan 2006). The top panel shows $n_{\text {sats }}$ as a function of $z_{\text {reion }}$ when $M_{\mathrm{t}}$ is set such that star formation begins once the subhalo reaches a virial temperature $T_{\text {vir }}=8 \times 10^{3} \mathrm{~K}$. This line asymptotes to $n_{\text {sats }}=7$ because we allow star formation to resume after reionization for halos that shock heat to temperature $T_{\mathrm{vir}}=10^{5} \mathrm{~K}$. Virial temperatures are calculated using the relation

$$
\begin{array}{r}
M=10^{6} \mathrm{M}_{\odot}\left[\left(\frac{T_{\mathrm{vir}}}{1800 . \mathrm{K}}\right)\left(\frac{21}{1+z}\right)\left(\frac{1.22}{\mu}\right)\right]^{3 / 2} \times \\
\left(\frac{0.3}{\Omega_{M, 0}}\right)^{1 / 2}\left(\frac{0.7}{h}\right),
\end{array}
$$

where $\mu=0.57$ for $T_{\mathrm{vir}}<1.5 \times 10^{4} \mathrm{~K}$ and 1.22 for $T_{\mathrm{vir}} \geq$ $1.5 \times 10^{4} \mathrm{~K}$ (Haiman \& Bryan 2006). The model referred to as "all galaxies" in this plot indicates all subhalos capable of hosting a galaxy. The galaxies are not defined in terms of a magnitude limit, but weather they reached a mass scale (left

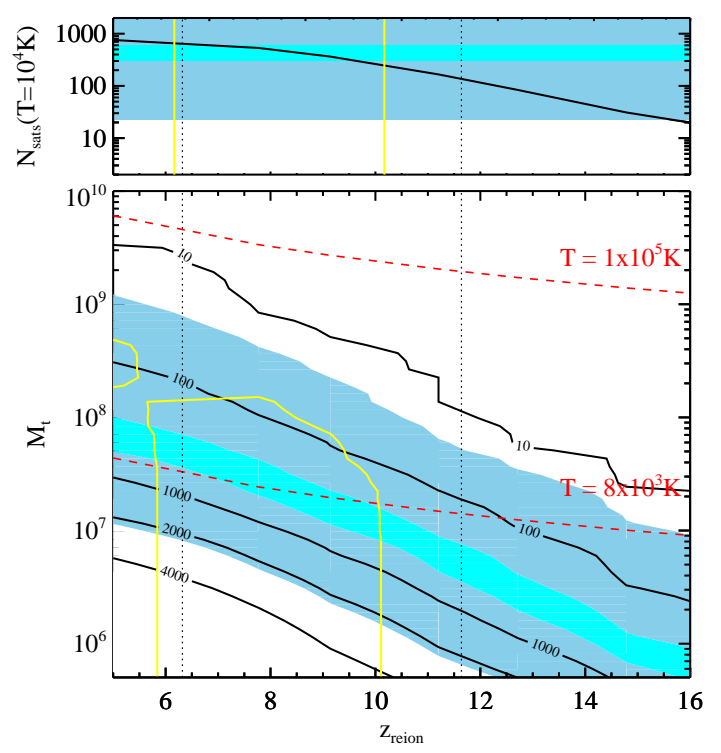

FIG. 3.- Bottom: The number of surviving satellite galaxies in the via Lactea IIsimulation assuming that subhalos had to grow above a threshold mass $M_{\mathrm{t}}$ by $z_{\text {reion }}$ in order to host a galaxy. The dashed vertical lines show the 95\% confidence intervals for the reionization epoch of Milky Way mass halos. The dashed red lines denote constant virial temperatures, where $8 \times 10^{3} \mathrm{~K}$ is the expected temperature for allowing star formation through $\mathrm{HI}$ cooling. The the light and dark shaded regions show the observational constraints on the both the maximal and most likely distribution in the number of satellite galaxies from Tollerud et al. (2008). The yellow contours denote the $95 \%$ confidence levels for constraining $z_{\text {reion }}$ and $M_{\mathrm{t}}$ by matching with the $v_{\max }$ function of Figure 7 Top: The number of satellite galaxies as a function of $z_{\text {reion }}$ assuming that $M_{\mathrm{t}}$ follows the $T=8 \times 10^{3} \mathrm{~K}$ curve. The dotted lines show the $95 \%$ distribution for $z_{\text {reion }}$ for Milky Way mass halos, and the yellow lines show the $95 \%$ confidence range in $z_{\text {reion }}$ at this $M_{\mathrm{t}}$. Again, the light and dark cyan regions show full and most likely range for the number of Milky Way satellite galaxies.

as a variable in this model) above which star formation was allowed to proceed. As a consequence, many such galaxies are expected to be extremely low luminosity, well below current detection limits. Additionally, the model is simply a function of the subhalo mass at times prior to accretion and does not track effects such as tidal disruption.

The most striking feature of this plot is the size of the variation in $n_{\text {sats }}$, which can differ by roughly an order of magnitude at fixed $T\left(M_{\mathrm{t}}\right)$ as $z_{\text {reion }}$ is varied within the $95 \%$ distribution or as $M_{\mathrm{t}}$ changes from $T_{\mathrm{vir}}=10^{3}-10^{4} \mathrm{~K}$. For a constant $M_{\mathrm{t}}$, the number of satellites has a roughly exponential dependence on $z_{\text {reion }}, n_{\text {sats }} \propto e^{-0.75 z_{\text {reion }}}$. Again, there is an imposed minimum value of $n_{\text {sats }}=7$ for any value of $z_{\text {reion }}$, set by the assumption that photoionized gas can cool and form stars when a halo shock heats to $T_{\mathrm{vir}}=10^{5} \mathrm{~K}$; seven of the via Lactea II subhalos reach this temperature at some point in their histories.

The shaded region of Figure 3 shows the observational constraints on the number of satellite galaxies from the work of Tollerud et al. (2008). The lighter regions shows the extreme case of this analysis, where the Milky Way has between 23 (currently observed) and 2261 satellites galaxies, while the darker region shows what their work considers the more likely prediction of $300-600$ satellites. When all this data is combined, the consistency is rather surprising. The region spanned by the upper and lower 95th percentile for $z_{\text {reion }}$ of Milky Way mass halos, as well as the $M_{\mathrm{t}} \approx 10^{6} \mathrm{M}_{\odot} h^{-1}$ from 
Abel et al. (2002) and the $T_{\text {vir }}=8 \times 10^{3} \mathrm{~K}$ falls entirely within the constraints of Tollerud et al. (2008). It is important to note that these results rely on simulations using completely different sets of physics. The limits on $M_{\mathrm{t}}$ are set from hydrodynamical simulations as discussed in $\$ 2.2$, while the $z_{\text {reion }}$ and $n_{\text {sats }}$ limits come from $\mathrm{N}$-body simulations that model only collisionless physics.

One caveat that must be kept in mind when interpreting Figure 3 is that we have assumed the particular subhalo population of via Lactea II is representative of a typical Milky Way mass halo. While only a handful of such ultra-high resolution simulations have been conducted, it is already apparent that there is a wide distribution in the number of subhalos in halos of similar mass. In particular, currently the three most well resolved halos (Diemand et al. 2008; Stadel et al. 2008; Springel et al. 2008) contain a factor of 1.5-2 more subhalos than via Lactea II at a fixed mass threshold, and it is estimated that via Lactea II is among the $15 \%$ of objects with similar mass that have so few subhalos (Ishiyama et al. 2008). If this is the case, the number of satellites predicted in Figure 3 is potentially a factor of two too low for a typical Milky Way mass halo. However, it is unknown exactly where in the relatively wide distribution the Milky Way lies, particularly since the number of subhalos has been shown to correlate strongly with halo concentration and formation history (Zentner et al. 2005). In the remainder of this work, we assume that the subhalos in via Lactea II are representative of the Milky Way, but this distribution, and the possible bias, should be kept in mind when detailed numerical results are given.

\subsection{Luminosity Function}

While Figure 3 shows that the total number of subhalos hosting satellite galaxies may be strongly dependent on the time of reionization, it is necessary to understand the properties of these affected halos, i.e., are they all low mass objects that we expect to host low-luminosity galaxies, or do they fill a larger range in satellite parameter space? In order to quantify the expected impact on observations, we must first impose the relevant observational cuts on our satellite distribution. For each subhalo, we calculate $r_{\text {sun }}$, the distance from a point $8 \mathrm{kpc}$ from the center of via Lactea II. Figure 4 shows this distribution as a function of magnitude for the model $z_{\text {reion }}=8$, $M_{\mathrm{t}}=3 \times 10^{7} h^{-1} \mathrm{M}_{\odot}$. The open red circles show magnitudes calculated using the abundance matching method (equation 11, and the filled green triangles use the population synthesis model. We then impose the cut defined by equation 3 above, shown as the black line. Because we expect this subset to best match the observational sample, this cut is imposed for all subsequent comparisons. While only affecting about $20 \%$ of our satellites, objects as bright as $M_{V}=-7$ are cut. The distributions of the Milky Way dwarfs are overplotted for reference. We also calculate the detection efficiency for each satellite galaxy based on its surface brightness according to equation 4 All galaxies passing out magnitude cut have $\epsilon \approx 1$, so we do not make any additional cuts based on estimated surface brightness.

Again, because the magnitudes set by the abundance matching method are not directly impacted by $z_{\text {reion }}$ and $M_{\mathrm{t}}$, the distribution of objects in $M_{V}-r_{\text {sun }}$ space in not strongly impacted as these parameters are varied. In particular, adjusting these parameters only results in the presence or absence of objects with low $M_{V}$ as low mass subhalos gain or lose the ability to host satellite galaxies. Individual objects will, however, have a significant dependence on magnitude in the SPS model be-

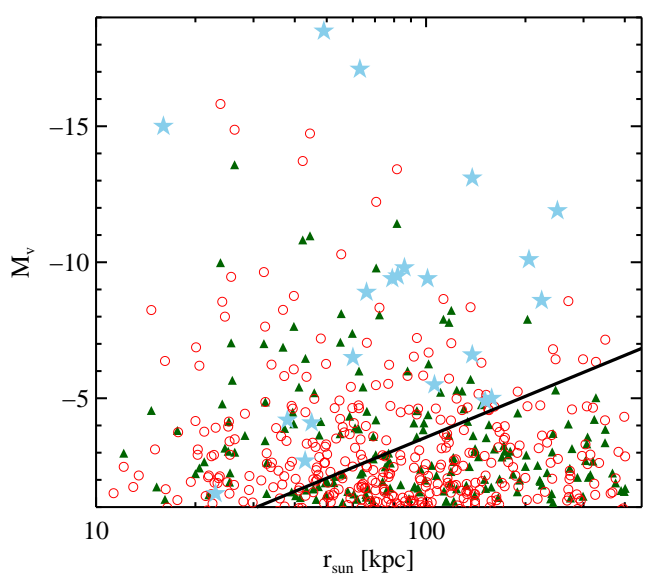

FIG. 4. - The distributions of satellite galaxy magnitudes as a function of distance from the sun in a model with $z_{\text {reion }}=9$ and $M_{\mathrm{t}}=3 \times 10^{7} h^{-1} \mathrm{M}_{\odot}$. The open red circles show magnitudes assigned using the abundance matching method (equation 10 and the filled green triangles have magnitudes assigned using the Bruzual and Charlot stellar population synthesis (SPS) code. The cyan stars show the distribution of the observed Milky Way satellites. The solid line shows the completeness depth of the SDSS survey as given by equation 3

cause adjusting these parameters impacts how long star formation is allowed to proceed for, impacting the amount of mass that can be converted into stars. In addition to forming new satellite galaxies, pushing $z_{\text {reion }}$ to later epochs also causes the existing satellites to brighten.

Figure 5 compares the luminosity functions from our model with observations. The thicker lines show magnitudes set by the abundance matching method, and the thinner lines by the SPS model. For this plot, we have fixed $M_{\mathrm{t}}$ to be set by the $T_{\text {vir }}\left(M_{\mathrm{t}}\right)=8 \times 10^{3} \mathrm{~K}$ relation and varied $z_{\text {reion }}=5,8$, and $12($ red dotted, green dashed, and blue dot-dashed lines). The black long-dashed line with points shows the observed luminosity function, while the cyan region shows the Poisson errors about this distribution.

Overall, both the abundance matching and SPS methods reproduce the observed luminosity reasonably well. The level of agreement for the abundance matching method in particular is rather impressive since the method 1) ignores all baryon physics such as star formation, 2) assumes all galaxies have average color, and 3 ) extrapolates number densities down to regimes where the method has not been tested and where small-scale processes may produce a significant amount of scatter in the $v_{\max }-M_{V}$ relation. Because the primary effect of changing $z_{\text {reion }}$ is to change the total number of satellite galaxies, this serves to change the overall normalization of the luminosity function while retaining the slope. Although not shown here, changing $M_{\mathrm{t}}$ for a fixed $z_{\text {reion }}$ has a similar effect.

The high level of agreement between our SPS model and observations comes from tuning the efficiency parameter, $\epsilon$, to match observations independently for all values of $z_{\text {reion. }}$. Thus, the three curves in Figure 5 have values $\epsilon=0.1,10$, and $300 \mathrm{M}_{\odot} / \mathrm{yr}$, which were selected for no physical reason other than to match the luminosity function. It is, however, interesting to note that the faint-end slope of this model almost perfectly matches the observations. The underprediction of luminosities at the bright end can potentially be explained by residual, ongoing star formation. At least one of the classical Milky Way Dwarfs, Leo I, shows signifi- 


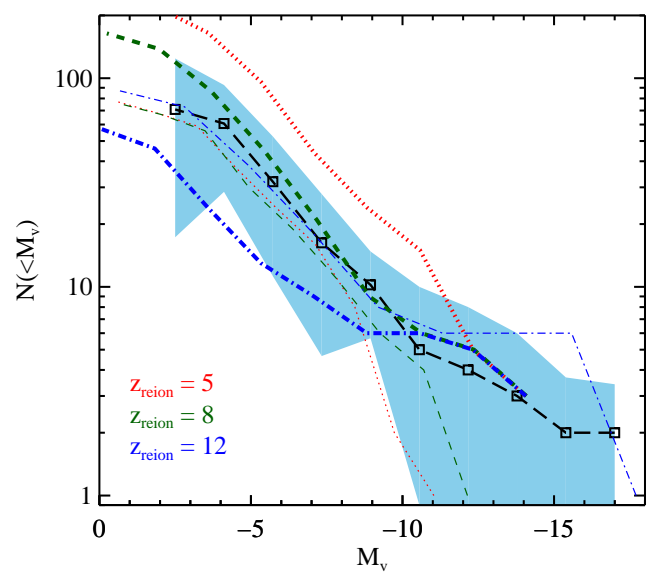

FIG. 5. - Luminosity functions for observations and model predictions. The long-dashed line shows the observed Milky Way satellite luminosity function corrected for sky coverage and depth effects, while the cyan swath represents the statistical error. The red dotted, green dashed, and blue dotdashed lines represent reionization models of varying $z_{\text {reion }}=5,8$, and 12 , respectively. $M_{\mathrm{t}}$ is set using the virial temperature, $T_{\mathrm{vir}}\left(M_{\mathrm{t}}\right)=8 \times 10^{3} \mathrm{~K}$. The thicker set of lines shows predicted luminosity functions using an extrapolated abundance matching method to assign luminosities to the galaxies. The thinner set of lines use a stellar population synthesis model to predict the luminosities.

cant evidence - while Fornax shows slight evidence - for recent star formation after the epoch of accretion (Mateo 1998; Mateo et al. 2008). If we were to allow some such process in our 4 brightest objects the model will likely fit the observations significantly better.

Finally, we consider the ration of mass to luminosity in the top panel of Figure 6 Here, we plot the mass to light ratio of the via Lactea II subhalos using their virial masses at time of accretion and luminosities assigned from the abundance matching method for $z_{\text {reion }}=8$ as red circles. Solid circles represent objects within the SDSS magnitude limit, $r_{\text {sun }}<R_{\text {comp }}\left(M_{V}\right)$, where $R_{\text {comp }}$ is given by equation 3 . Open circles are satellites outside this limit. This model naturally reproduces a wide range of $M / L$ rations, spanning from $10^{3}$ to $10^{6}$, with a clear trend with luminosity, $M / L \propto L^{-0.52}$. Green triangles represent luminosities calculated using our SPS method. This method reproduces a similar trend with luminosity but with a larger dispersion. We have included measurements from the Milky Way as the cyan stars. In order to model the masses of these objects, we took the $M_{0.3}$ values for the mass within $300 \mathrm{kpc}$ published in Strigari et al. (2008) and converted those to subhalo masses using their published relation $M_{0.3}=10^{7} \mathrm{M}_{\odot}\left(M_{v i r} / 10^{9} \mathrm{M}_{\odot}\right)^{0.35}$, where $M_{\text {vir }}$ is the virial mass of the subhalo at the time of accretion. We must caution that this relation is expected to be dependent on cosmology and ignores all scatter. The observations are remarkably well matched by our model with excellent agreement for all but the most most luminous galaxies. In the lower panel of Figure 6 we consider this data in a different way by plotting $M_{0.3}$ as a function of luminosity. Here, we take the data directly from Strigari et al. (2008) and convert the via Lactea II subhalo masses to $M_{0.3}$ using the above formula. While the numbers are in general agreement, the abundance matching model (red circles) shows a clear trend with luminosity, $M_{0.3} \propto L_{V}^{0.17}$, as opposed to the observations (cyan stars) which indicate more of a common mass scale. The trend in our model results directly from the abundance matching

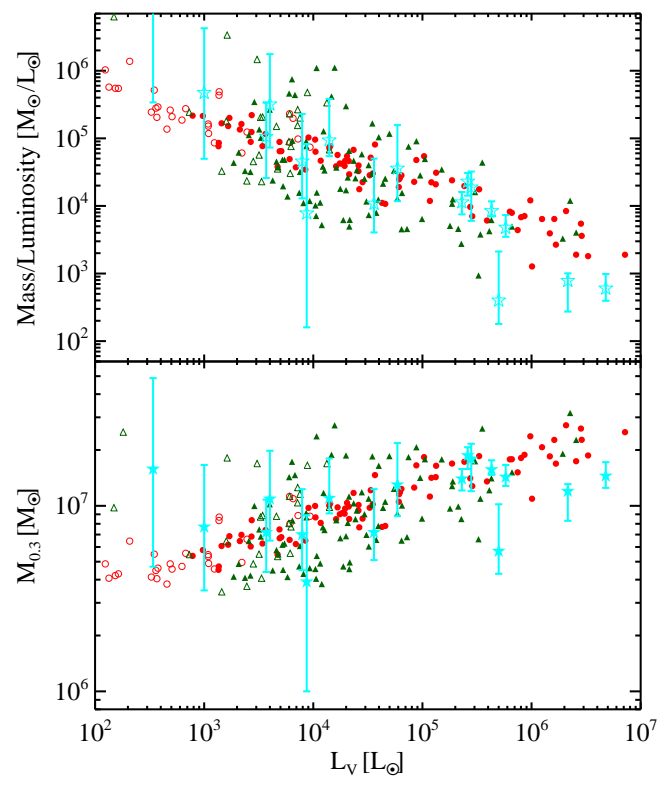

FIG. 6. - Top Panel: The ratio of subhalo mass at the time of accretion to luminosity for our abundance matching model (red circles) with $z_{\text {reion }}=8$. Filled circle represent halos that are within the SDSS completeness radius, equation 3 while open circles are outside this distance. Cyan stars represent Milky Way satellites. In order to make this comparison, we used the values for mass within $300 \mathrm{kpc}$ at the present epoch, $M_{0.3}$, published in Strigari et al. (2008) and converted them to subhalo masses using their published relation for the average $M_{D M}\left(M_{0.3}\right)$ calibrated to N-body simulations. Bottom Panel: The relation between $M_{0.3}$ and luminosity for our abundance matching model and observations. Here, we have converted the masses of the via Lactea II subhalo to $M_{0.3}$ values using the formula provided by Strigari et al. (2008).

method, which assigns luminosities to subhalos satellite based on $v_{\max }$ at the time of accretion. The addition of scatter into either the $L_{V}\left(v_{\max }\right)$ or $M_{0.3}\left(M_{v i r}\right)$ relations, which we expect at these low mass scales, can help to flatten this trend slightly and bring it more in line with observations. The SPS model (green triangles) also produces a similar trend with luminosity albeit with a significantly larger scatter, making the slope of the $M_{0.3}\left(L_{V}\right)$ relation consistent with zero. Previous studies of simulations have observed a similar trend (e.g., Maccio' et al. 2008; Li et al. 2008; Koposov et al.2009) using semi-analytic modeling of galaxies and/or subhalo distributions.

\subsection{Circular Velocity and Radial Distributions}

We next consider the mass distribution of the satellite galaxies hosting halos. Figure 7 shows the changes in the $v_{\max }$ distribution for satellites as $z_{\text {reion }}$ is varied, given a threshold mass of $T_{\text {vir }}\left(M_{\mathrm{t}}\right)=8 \times 10^{3} \mathrm{~K}$ as in Figure 5. Here, the solid black line shows the distribution for all via Lactea II subhalos, while the red dotted, green dashed, and blue dot-dashed show the distributions from our model for three values of $z_{\text {reion }}=5,8$, and 12 . Because the abundance matching method was more successful than the SPS model in reproducing the observed luminosity function without the need to tune any parameters, we only include satellites that pass the radial cut of equation 3 using the abundance matching criteria. As can be seen, an earlier $z_{\text {reion }}$ suppresses the distribution of subhalos with all values of $v_{\max }$, although the effect is more pronounced for low mass halos. Still, this suppression is present even for $v_{\max } \gtrsim 20 \mathrm{~km} / \mathrm{s}$, where most of the classical dwarfs live. This indicates that $z_{\text {reion }}$ can effect satellite galaxies of all masses and luminosities. 


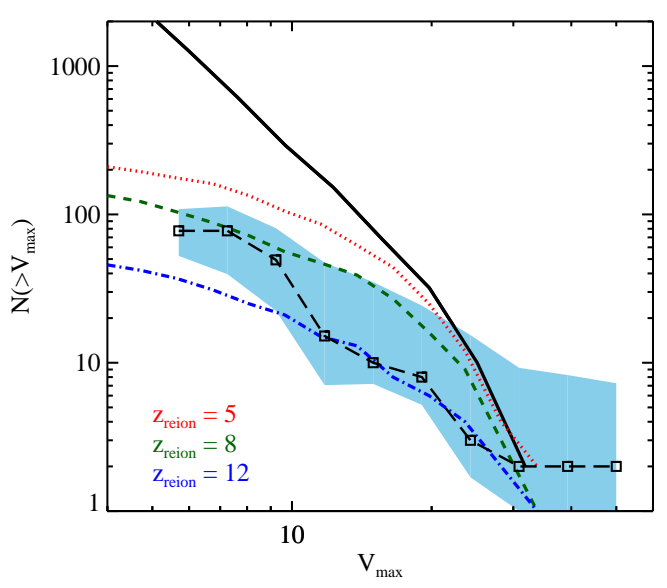

FIG. 7. - The peak circular velocity functions for subhalos hosting satellite galaxies. The solid line shows the velocity function for all via Lactea II subhalos. The red dotted, green dashed, and blue dash-dotted lines show the distribution for subhalos hosting satellite galaxies for $z_{\text {reion }}=5,8$, and 12 with $T_{\mathrm{vir}}\left(M_{\mathrm{t}}\right)=3 \times 10^{8} \mathrm{~K}$, as in Figure 5 The long dashed black line shows observed distribution for Milky Way satellites, corrected for sky coverage and detection efficiency. The cyan bands show combined Monte Carlo and statistical errors.

The long-dashed black line with data points again represents the observations. The $v_{\max }$ values for the satellites, including errors, were calculated using the method of Strigari et al. (2007b a) using kinematic data taken from the literature (Walker et al. 2007; Simon \& Geha 2007). The line was calculated using the 22 observed satellites and correcting them for SDSS sky coverage and detection efficiency (Koposov et al. 2008). The cyan region denotes errors on this curve and were calculated using a Monte Carlo approach. In this approach, published errors are used where possible; where no robust errors are published, the average error distribution is mapped onto the remaining SDSS dwarfs. While this process does not produce uncorrelated error bars, it should be significantly more robust than simply using statistical uncertainties. We should also note that, because the reconstruction of $v_{\max }$ from observations gives a very strong lower bound but only a weak upper bound, the errors on the lowest points are probably underestimated because very few satellites will scatter into this bin as we create a Monte Carlo representation of the distribution. These systematic errors were combined with statistical errors assuming a Poisson distribution.

Figure 8 further explores the impact of varying $z_{\text {reion }}$ on the properties of the satellites and the subhalo hosts by considering the radial distribution within the halo. The lines represent the same populations as in Figure 7]. Here, the errors on the observations are purely Poisson. Again, there is strong trend for an early reionization epoch to suppress the abundance of satellite galaxies at all radii, but, in part because the data is rather noisy, it remains easy to match the model to the observations.

Finally, using our magnitude-limited sample of satellites from the abundance matching method, we can attempt to put constraints on $z_{\text {reion }}$ and $M_{\mathrm{t}}$. We calculate the $\chi^{2}$ errors between the $v_{\max }$ distribution for observations and our models and plot the $95 \%$ confidence levels as the yellow contour in Figure 3 We use the $v_{\max }$ distribution to generate this constraint because it is less sensitive to the modeling of the magnitudes than the luminosity function is, with the modeling only coming into play when making the SDSS magnitude-

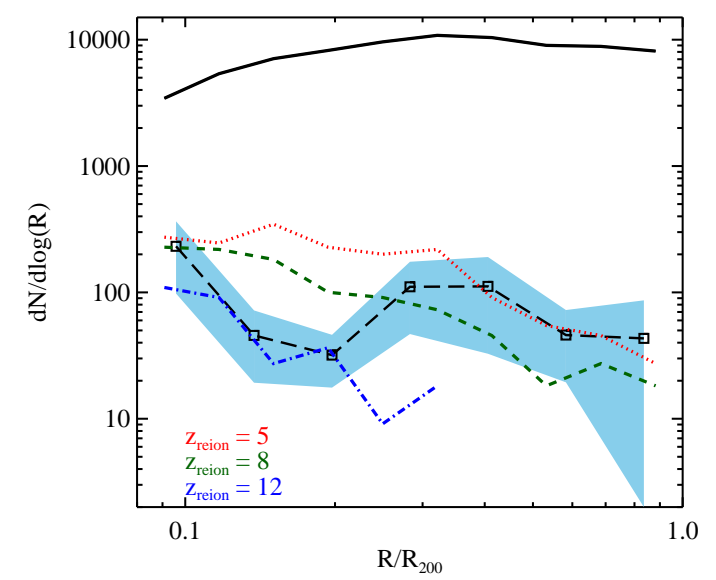

FIG. 8. - The radial distribution subhalos hosting satellite galaxies in via Lactea II. The lines represent the same populations as in Figure 7

limit cut. Because the Monte Carlo method underestimates the error on the lowest $v_{\max }$ point in Figure 7 , we exclude it from our constraints. The general insensitivity of our constraints to the value of $M_{\mathrm{t}}$ comes from our use of $v_{\max }\left(z_{\text {reion }}\right)$ in setting the magnitude of a galaxy. While lowering the threshold for star formation from $\sim 10^{8}$ to $\sim 10^{9} h^{-1} \mathrm{M}_{\odot}$ significantly increases the number of subhalos hosting galaxies, most of these objects are quite dim, with $M_{V} \gtrsim-4$, and are therefore cut from our observable sample (see Figure 4). If we impose the condition that galaxy formation begins when a halo reaches virial temperature $T_{\text {vir }}\left(M_{\mathrm{t}}\right)=3 \times 10^{8} \mathrm{~K}$ we can constrain the time of reionization to $z_{\text {reion }}=8_{-2}^{+3}$, with $540_{-340}^{+100}$ satellites. Note that these errors assume that the dark matter substructure of the Milky Way is identical to that of the via Lactea II halo; if the Milky Way is more typical for its mass it may have a larger total number of observable satellites. A further success of this model is that, when we constrain $z_{\text {reion }}$ using the $v_{\max }$ function, we naturally reproduce both the observed luminosity function and radial distribution, as shown by the green dashed lines in Figures 5 and 8 . We should, however, caution again that much of this depends on the particular realization that is the via Lactea II halo, and that more statistics will be necessary for a more robust prediction.

\section{COMPARISON WITH PREVIOUS WORK}

Our result that the number of satellite galaxies is strongly dependent on the redshift of reionization is at odds with some previous studies, including the work of Somerville (2002), Kravtsov et al. (2004b), and Orban et al. (2008), although these studies primarily focused on the classical satellite galaxies in the Milky Way. The primary difference in our models rests on the assumption of how reionization effects the presence of cold gas. These previous studies have used the model of Gnedin (2000) to calculate the amount of cold gas in a halo of mass $M$. They calculate a filtering mass, $M_{f}(z)$, the mass of a halo that looses half its baryons compared to the universal baryon fraction in the presence of a photoionizing UV background. This mass is related to the baryon fraction of a halo of mass $M$ via the relation

$$
f_{\text {gas }}(M, z)=\frac{f_{\text {baryon }}}{\left[1+0.26 M_{f}(z) / M\right]^{3}},
$$

where the details for calculating the filtering mass, $M_{f}$, are given in Gnedin (2000) and Appendix B of Kravtsov et al. 
(2004b).

The crucial point in using the values of $f_{\text {gas }}$ from Gnedin (2000) lies in how these fractions are related to the amount of cold gas available for star formation. There are two bracketing possibilities: either the gas is spread out over the entire halo and is hot, in which case the star formation rate is zero, or, there is a small clump of cold gas in the center, with a mass given by $f_{\text {gas }} M_{\text {halo }}$, that can form stars. Our interpretation, in which the gas is assumed to be hot for $f_{\text {gas }} \ll 1$, is consistent with the first possibility, while these previous studies have implicitly assumed that all the gas is cold.

There are several reasons to favor the hot gas scenario. Most importantly, Gnedin (2000) did not distinguish between hot and cold gas when calculating the gas fraction. The assumption that the gas is cold is only valid for halos that had collapsed before the reionization epoch, and were then subject to photo evaporation due to the UV background. Such cold gas would only survive for one photo evaporation time, which, according to the numerical simulations of Iliev et al. (2005), is likely to be less than $500 \mathrm{Myr}$, corresponding to $\Delta z<2$ at $z<6$. After such photo evaporation, the halo could only accrete hot gas, which would be unlikely to form stars given its low density and long cooling time. Moreover, due to the exponential growth of the abundance of halos with masses $\sim 10^{8} M_{\odot}$ during reionization, it is likely that most halos present after reionization were only just collapsing, and thus did not have any cold gas capable of forming stars in the first place. We therefore interpret the gas fractions $f_{\text {gas }} \ll 1$ reported by Gnedin (2000) after reionization as corresponding to hot gas when $f_{\text {gas }} \ll \Omega_{b} / \Omega_{m}$.

Another related caveat in using $f_{\text {gas }}$ to model the dependence of star formation history on reionization is that the value of $f_{\text {gas }}$ reported by Gnedin (2000) is a mean value, for all halos in the box, regardless of whether they are in ionized regions or not. In reality, those halos that were reionized earlier have lower gas mass fractions than those that were reionized later. Using the average value of $f_{\text {gas }}$ for all halos underestimates the sharpness of the transition for halos that were ionized at a given time. Because our reionization redshift is defined for a given halo (as opposed to a universal time for the universe), we expect the transition in gas fraction to be much sharper than that given by averaging over all halos, each with

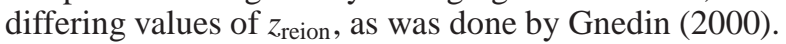

The difference between these scenarios - where $f_{\text {gas }}$ represents the fraction of hot in a halo consistent with our abundance matching and SPS models as opposed to the fraction of cold gas available for star formation - is illustrated by Figure 9. which shows the evolution of $f_{\text {gas }}(M, z)$, the material available for star formation, with redshift. The upper and lower pairs of lines in the figure represent halos with mass $M=10^{8} h^{-1} \mathrm{M}_{\odot}$ and $10^{6} h^{-1} \mathrm{M}_{\odot}$. The solid and dashed lines represent models where the universe reionizes at different epochs, $z=5$ and12, respectively. At high redshift, halos have a gas fraction equal to the universal baryon fraction. As time evolves, the $f_{\text {gas }}$ of the average halo decreases rather slowly, due to photoionization heating as halos begin to be exposed to the UV background. Changing the redshift of reionization affects the rate of transition from the cold to the hot phase, but the overall shape of the transition is preserved. As we emphasized above, because the average over all halos was used to calculate the filtering mass, this is likely an accurate description of how reionization effects the gas content of an average halo of mass $M$ given a universal redshift epoch. We

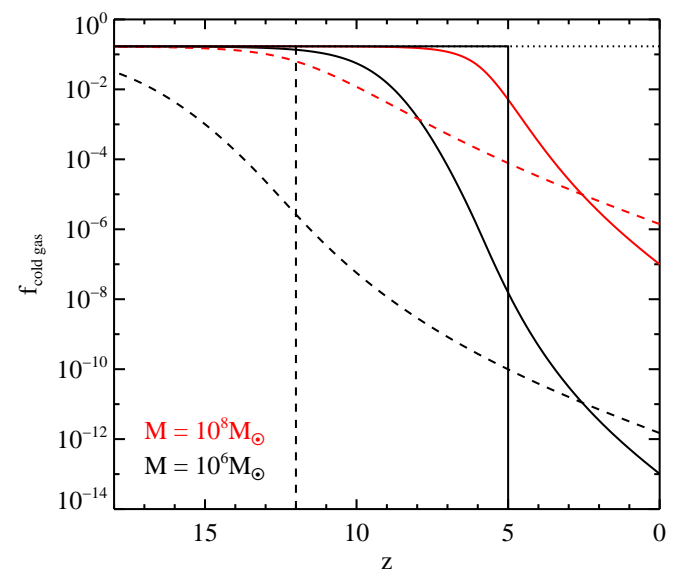

FIG. 9. - The fraction of mass in the form of cold gas as a function of time. The solid and dotted lines are calculated using universal reionization epochs $z=5$ and 12, respectively. The lower (black) and upper (red) pairs of curves show the predictions from Gnedin (2000) for halos of mass $M_{D M}=10^{6} h^{-1} \mathrm{M}_{\odot}$ and $10^{8} h^{-1} \mathrm{M}_{\odot}$, respectively. The vertical lines represent the model used in this work, where the reionization time of the subhalo is $z_{\text {reion }}=6.5,11.5$ for the solid and dashed lines. The horizontal dotted line shows the universal baryon fraction.

contrast this with the model used for this work, shown by the vertical lines indicating a sharp cutoff in the presence of cold gas at the time at which the halo reionizes is mass independent. While we acknowledge that we are ignoring effects such as atomic recombination and additional cooling for high mass objects that should not quite reduce the cold gas fraction to zero after reionization, we expect that such a sharp transition from the cold to hot phase more accurately describes the evolution of an individual halo.

The difference in the resulting star formation histories in the two scenarios discussed above is dramatic. To show this explicitly, we have re-run our population synthesis model using the more gradual star-formation squelching model of equation 6 instead of an abrupt squelching for setting $f_{\text {coldgas }}$ in equation 2, although we still assume an abrupt end to star formation when the subhalo is accreted onto the host halo. The result, shown in Figure 10, is that the luminosity function of the satellite galaxies becomes largely independent of the reionization epoch, in agreement with these previous studies. Here, the thin lines represent our model with an instantaneous gas heating, while the thick lines show the gradual turn off from equation 6 The red dotted, green dashed, and blue dot-dashed show the effect of changing the reionization time, $z_{\text {reion }}=5,8,12$. We should note that, unlike in Figure 5, we did not tune the parameters $\alpha$ and $\epsilon$ of equation 2 to reproduce the observational sample at all values of $z_{\text {reion }}$, but just set the parameters to fit the $z_{\text {reion }}=9.6$ model. Additionally, this plot does not include any observational magnitude cuts, equation 3. While the instantaneous-squelching model shows dramatically different luminosity functions as $z_{\text {reion }}$ is changed, the gradual cutoff model is remarkably stable. This is because, even though the rate at which gas heats varies with reionization time, the gradual turnoff causes roughly the same amount of stellar mass to form in an average halo regardless of the reionization time. The average stellar mass, $\left\langle\log \left(M_{\text {stellar }}\right)\right\rangle$, changes by less than $5 \%$ as $z_{\text {reion }}$ varies from 12 to 5 . For our rapidly truncating model, however, the amount of gas converted into stars clearly depends on the time of reionization. This raises an important point, in that while there is some dis- 


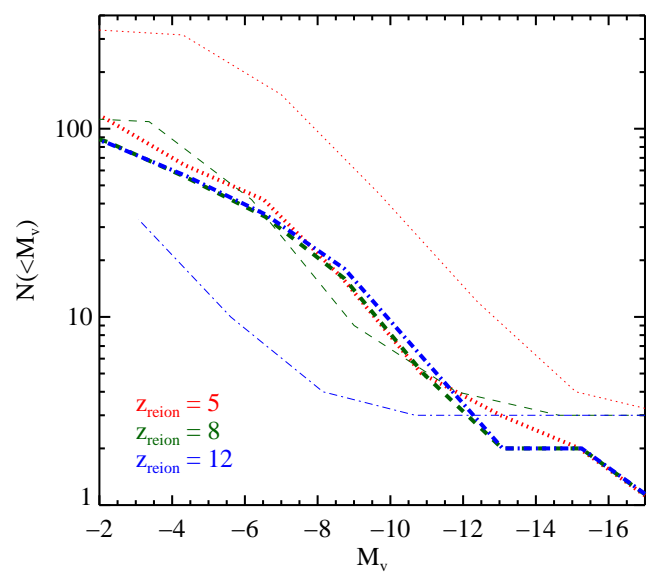

FIG. 10.- The luminosity functions from our SPS model for both the case of an instantaneous gas heating (thin lines) and a more gradual turnoff as given by equation 6 (thick lines). Colors and line-styles represent different epochs of reionization as in Figure 5

agreement as to the impact the time of reionization has on the satellite galaxy population of a halo, the rate at which star formation is squelched can also have a significant impact on the population.

In order to understand why the luminosity function does not vary with $z_{\text {reion }}$ in this model, it is necessary to look at the magnitudes of individual galaxies. The most massive subhalos in via Lactea II are larger than the filtering mass, $M_{f}$, and are therefore unaffected by changes in the reionization epoch. In order to understand why the dim end of the luminosity function has such a weak dependence on the reionization epoch, we note that, as pointed out in Koposov et al. (2009), halos in this model with a gradual turnoff of star formation create a large number of stars after $z_{\text {reion }}$. Additionally, as seen in Figure 9 there is a transition epoch around $z=3$ between suppression and enhancement of $f_{\text {gas }}$ for early and late reionization times. At early epochs, an early $z_{\text {reion }}$ suppresses the amount of gas in a halo of a given mass relative to a late reionization because of the additional energy input to the system. However, at late times, the early $z_{\text {reion }}$ actually causes an enhancement relative to later reionization because the expansion of the universe since reionization causes adiabatic cooling. This transition is roughly independent of mass. Thus, there are two regimes: Subhalos that accrete onto the main halo early, before $z=3$, must get dimmer as $z_{\text {reion }}$ increases, because star formation is suppressed at all epochs. However, objects that accrete recently pass through a phase where earlier reionization enhances star formation, potentially allowing such satellites to brighten with.

This trend between star formation and time is seen in Figure 11. which plots $\Delta\left(M_{V}\right)$, the difference in magnitude for individual satellite galaxies between early $\left(z_{\text {reion }}=12\right)$ and late $\left(z_{\text {reion }}=5\right)$ reionization, as a function of accretion time within the context of a gradual star formation turnoff. Positive values represent satellites that brighten with early reionization, while negative values represent objects that get dimmer with early reionization. There is a clear trend with $z_{\text {accretion }}$ : Prior to $z=3$, all objects have negative values for $\Delta\left(M_{V}\right)$, as expected from Figure 9. while objects that accreted more recently have both positive an negative values, with the most positive values (strongest brightening from earlier reionization) coming from the most recently accreted satellites. Thus, while $z_{\text {reion }}$ does effect the magnitude of a given satellite in the context of

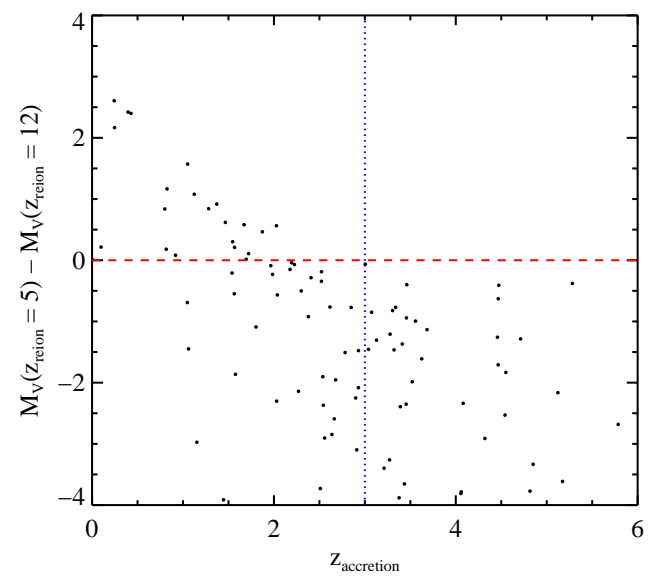

FIG. 11. - The change in magnitude for satellite galaxies between late $(z=12)$ and early $(z=5)$ zreion using the model motivated by Gnedin (2000), where the amount of cold gas is given by equation 6 Galaxies that have been accreted more recently are brighter for earlier $z_{\text {reion }}$ due to the boost in star formation from adiabatic cooling. The opposite trend is present for galaxies that are accreted earlier.

a model with a gradual star formation turnoff, there are two competing effects that cause the luminosity function to be unchanged. This explains the differences between our results and those of Somerville (2002) who concluded that $z_{\text {reion }}$ has no effect on the satellite galaxy luminosity function. Hydrodynamical simulations also tend to disfavor this scenario. As shown in Abel \& Haehnelt (1999) and Sokasian et al. (2002), quasar activity around $z \sim 3-4$ would have resulted in an epoch of HeII reionization, something not considered in the calculations of Gnedin (2000). This would have resulted in additional heating of the gas at these epochs, likely destroying both the enhanced recent star formation activity due to early reionization and independence of the satellite galaxy luminosity function on the epoch of reionization.

We also reach different conclusions than the recent work of Koposov et al. (2009) as to whether an abrupt or a gradual star formation truncation more accurately matches observations. In particular, they note a bimodality in the luminosity function for an abrupt cutoff to star formation. Their model differs from ours, however, in the key respect that they assume a halo converts a constant fraction of its gas mass into stars, resulting in stellar mass $M_{*} \propto M_{D M}$, where $M_{D M}$ is the dark matter mass at either reionization or $z=0$, depending on the mass of the halo. This results in a low-luminosity population of galaxies unable to form stars after reionization, and a higher luminosity population that can form stars. If we adopt such a model and apply it to the via Lactea II subhalos we reproduce a similar set of populations, with a population of dim satellites in a very narrow luminosity range separated from a brighter population made up of the 7 most massive subhalos that are able to sustain ongoing star formation after the reionization epoch. As Koposov et al. (2009) note, this is a poor match to the observations. However, in this work we adopted a more physically motivated model where the star formation rate depends on the halo mass, resulting in $M_{*} \propto \int M_{D M}^{\alpha} d t$ (e.g., Juneau et al. 2005; Noeske et al. 2007; Zheng et al. 2007; Conroy \& Wechsler 2009), where we integrate over the time between when the halo first crosses $M_{\mathrm{t}}$ and the reionization epoch. Changing the parameter $\alpha$ directly allows us to adjust the slope of the luminosity function and merge the two populations into a single, continuous distribu- 
tion. In particular, the value $\alpha=2$, measured from high mass objects (Conroy \& Wechsler 2009; Drory \& Alvarez 2008) provides a close fit to the data, Figure 5. Thus, we conclude that a sharp cutoff of star formation can closely match the observational data.

\section{DISCUSSION AND CONCLUSIONS}

\subsection{Additional Constraints and Physical Processes}

Here we briefly discuss additional physics processes not addressed earlier. One observable constraint on our model is the presence of fossil galaxies - objects that have had no star formation since reionization. Current observational constraints indicate that all satellites brighter than $M_{V} \sim-8$ have had some sort of star formation in the past $8 \mathrm{Gyr}$ (see the review of Tolstoy et al. 2009, and references therein), but that dimmer objects such as Hercules may have had all their stars formed during a single event (Koch et al. 2008). Our base model predicts a large number of fossil galaxies. As noted in section 2.2 , there are only seven "non-fossil" galaxies, whose mass reaches our post-reionization threshold of $10^{5} \mathrm{~K}$. The number of fossil galaxies in our model, however, is highly sensitive to the exact value of this threshold, while the overall abundance of satellite galaxies is not. The full impact will be explored further in a companion paper, but lowering the second cooling threshold from $10^{5} \mathrm{~K}$ to a few $10^{4} \mathrm{~K}$ can match the fossil constraints while not significantly impacting the predicted observed luminosity function.

Another limitation of our model as presented is our incomplete treatment of tidal disruptions on the satellite galaxy population. While the high resolution via Lactea II simulation automatically models the impact of tidal stripping on the dark matter halo, we have ignored the further impact on the stellar population. This is equivalent to the assumption that, once formed, a satellite galaxy remains undisrupted unless its host dark matter halos is completely disrupted, merging with the central object or dropping below the resolution limit of the simulation. Recent studies of Maccio' et al. (2009) and Koposov et al. (2009) have indicated that this may have a significant impact on the $z=0$ satellite luminosity function. To test this, we consider the criteria of Taylor \& Babul (2004) and Maccio' et al. (2009) for disruption, assuming that a satellite galaxy is destroyed when the tidal mass of its host dark matter subhalo is less than $M_{\text {bind }} \equiv M\left(<f_{\text {dis }} r_{\text {bind }}\right)$, where $r_{\text {bind }}$ is the radius of the subhalo, at infall within which the total energy is positive and $f_{d i s}$ is a free parameter that they set to $f_{\text {dis }}=0.1$. Taylor \& Babul (2004) calculate $r_{\text {bind }}=0.77 r_{s}$, where $r_{s}$ is the scale radius for an NFW profile. In order to estimate $M_{\text {bind }}$, we adopt the mean relation for concentration given mass and redshift, $c(M, z)=9\left(M_{v i r} / M_{*}\right)^{-0.13}$ in a $\Lambda$ CDM universe from Bullock et al. (2001). Using this, we see that, in our best fit model with $z_{\text {reion }}=8$, roughly $20 \%$ of our satellite galaxies are effected at all magnitudes. A further investigation of this process is needed, but it appears that there may be a significant degenracy between tidal disruption and reionization redshift when attempting to match the luminosity function.

\subsection{Future Work}

The analysis in this paper opens the door for a significant number of future studies. In particular, better statistics from both simulations and observations are necessary to distinguish between the models discussed here and confirm the result that $z_{\text {reion }}$ has a significant impact on the satellite galaxy population of a Milky Way mass halo.

On the simulation side, we need further analysis of both large scale and high mass-resolution simulations. By providing excellent statistics, large scale simulations of regions from 100-1,000 $\mathrm{h}^{-1} \mathrm{Mpc}$ will yield a significant amount of information about a wide range of properties of Milky Way mass halos, including environmental effects on both the reionization time and subhalo population as well as any correlations between the two. Clearly, there should be some correlation between clustering or the proximity to groups and clusters with $z_{\text {reion }}$ since such massive objects are the earliest sources of photoionizing radiation. Additionally, studies of halo assembly bias (e.g., Gao et al. 2005; Wechsler et al. 2006; Hahn et al. 2008) have shown correlations between halo assembly history, clustering, and substructure population. It is therefore likely that these properties will also correlate with the reionization time. Understanding such relations could allow us to measure the environment of the local group to both more robustly understand the subhalo population of the Milky Way and provide an alternate estimate for its reionization history. Such measurements could help confirm or refute the model presented here.

So far we have only applied our model to simulations of two single halos - via Lactea II and the original Via Lactea. In both simulations, we are able to reproduce the satellite luminosity and distribution functions to similar accuracy, albeit with $z_{\text {reion }}=8$ for via Lactea II and $z_{\text {reion }}=10$ for the original simulation. By studying additional, more highly resolved halos, we can both strengthen our predictions and accurately quantify the expected scatter in satellite galaxy population given $z_{\text {reion }}$ or an expected $n_{\text {sats }}$ for our model. Higher resolution will also allow us to accurately measure the distribution of mass down to scales of $300 \mathrm{kpc}$, easing comparisons with observations as in Figure 6 Indeed, these scales are just below the resolution limit in the most recent simulations (e.g., Diemand et al. 2008; Springel et al. 2008; Stadel et al. 2008). Finally, new high resolution simulations of the detailed hydrodynamics of star formation in the early universe can give us a better handle on how rapidly we expect reionization to truncate star formation by more accurately modeling the rate at which the gas in small halos is heated. Such simulations could also give independent predictions for the values of $\alpha$ and $\epsilon$ in equation 2 providing another way to test our model.

There are also a number of additional observational measurements that would help in understanding the validity of the models presented in this work. Most directly, deeper surveys will aid in quantifying the abundance of substructure by both extending the luminosity function to dimmer galaxies within the Milky Way, and providing measurements of other systems such as M31. Upcoming surveys such as DES, PanSTARRS, and LSST (Abbott et al. 2005; Kaiser et al. 2002; Ivezic et al. 2008) will map the distribution of galaxies at more than $3 \mathrm{dex}$ deeper than SDSS over the entire sky, potentially discovering hundreds of new satellites. These improved observations may also give us a better handle on mass measurements of the satellite galaxies. The depth will also allow us to potentially probe the bright end of the satellite luminosity functions for thousands of Milky Way mass galaxies at distances out to $\sim 60 \mathrm{Mpc}$. As with additional simulations, the statistics provided by these observations will provide significant discriminating power when applied to the models of this work.

Additionally, there is hope that detailed studies of the star formation history of the local satellite galaxies can help by 
directly measuring both the reionization history and rate at which reionization quenches star formation. Because the gradual star formation truncation given by equation 6 results in a significant amount of stars forming after $z_{\text {reion }}$, detailed modeling of star formation may be able to discriminate between models with rapid and gradual truncation of the star formation rate. Unfortunately for such modeling, any starburst activity occurring after a satellite accretes onto the host halo would greatly confuse the results. Because of this, we will likely have to concentrate on the dim end of the satellite luminosity function, restricting such studies to the Milky Way and potentially M31.

\subsection{Summary}

Following the recent work of Alvarez et al. (2008), we predict a broad range of reionization times for Milky Way mass halos, ranging from $z_{\text {reion }} \approx 6-12$. We find that the time of reionization can have a significant impact on the satellite galaxy population of a Milky Way halo. We investigate predictions for a simple model where, in order to cool gas and form stars, a subhalo must reach a threshold mass, $M_{\mathrm{t}}$, by $z_{\text {reion }}$, the time it reionizes. This model predicts a strong dependence of the satellite galaxy population on $z_{\text {reion }}$; we find that the number of satellites can vary by an order of magnitude for a fixed $M_{\mathrm{t}}$. This result is in contention with a number of previous studies which have shown minimal impact of $z_{\text {reion }}$ on the satellite population. The differing results are due to differing assumptions about the rate at which the UV background squelches star formation. Previous studies have used a gradual transition from the cold to hot gas phases, based the the work of Gnedin (2000) to predict the amount of cold gas available for star formation retained by a halo during the process of reionization. Instead, we interpret this calculation to be more indicative of the total amount of gas in the halo, and assume that it is rapidly heated to a hot phase so that star formation is very quickly stopped. Additionally, if the heating process causes a slow star formation truncation, we believe that the quasar HeII reionization at $z \sim 4-3$ will alter the star formation history such that $z_{\text {reion }}$ will more strongly impact the satellite luminosity function than previous studies have shown. While these two interpretations of rapid and gradual gas heating bracket the most extreme interpretations, their discrepancy indicates that further study into the exact heating rate is necessary since they predict completely different dependencies on $z_{\text {reion }}$.

Assuming a rapid heating of the gas from the photoionizing background, the reionization redshift $z_{\text {reion }}$ impacts the ability of subhalos of nearly all masses and radial distribution to host satellite galaxies, with a strong impact on the satellite galaxy luminosity function. In spite of the simplicity of the model, the results are consistent with both observations and detailed hydrodynamical simulations of stellar formation. Extrapolating the observed bright end relation between $v_{\max }$ and lumi- nosity down to satellite-sized objects, we are able to closely reproduce the luminosity function of the Milky Way tuning only the parameters of reionization. This is an additional indication of the robustness of the abundance matching method for assigning luminosities to dark matter halos.

Because it is minimally impacted by methods for assigning magnitudes to galaxies, we can use observations of the satellite $v_{\max }$ function to place constraints on the reionization epoch of the Milky Way. This observed distribution is best recovered for a reionization $z_{\text {reion }}=8_{-2}^{+3}$, slightly more recent than the "instantaneous" value of 11.2 from the WMAP5 data (Komatsu et al. 2008). This value is also in excellent agreement with the predictions from Weinmann et al. (2007) for the reionization redshift of a Milky-Way mass halo. This model predicts that the Milky Way should host roughly 540 satellite galaxies. Such a value for $z_{\text {reion }}$ also produces a good agreement for the radial distribution of satellites and the luminosity function when the abundance matching method is used. It must still be cautioned, however, that this result depends on assumption that the via Lactea II simulation is representative of the dark matter distribution in the Milky Way. We get similar agreement when we adopt a model where $S F R \propto M_{D M}^{\alpha}$, although it is important to note that this model has a tunable parameter. Still, it is able to reproduce the slope of the dim end of the luminosity function almost exactly and some late time star formation, consistent with observations, will help relieve the (slight) tension at the bright end. This is consistent with the recent work of Koposov et al. (2009). Our work lends further strength to the growing body of research that suggests that there really is no "missing satellite" problem for the Milky Way and that the next generation of surveys may allow us to understand the entire population of local satellite galaxies.

MTB would like to thank B. Gerke for many helpful discussions, as well as J. Diemand and collaborators for making the results of the via Lactea II simulation public. We would also like to thank Andrey Kravtsov, Oleg Gnedin, Nick Gnedin, James Bullock, Michael Kuhlen, and Charlie Conroy for many useful comments on the manuscript. MTB and RHW also thank their collaborators on the LasDamas project for critical input on the Gpc simulation, which was performed using the Orange cluster at SLAC. This work was partially supported by NASA ATFP grant NNX08AH26G, NSF AST0807312, and NSF AST-0908883. RHW was supported by a Terman Fellowship at Stanford University. LES acknowledges support for this work by NASA through Hubble Fellowship grant HF-01225.01 awarded by the Space Telescope Science Institute, which is operated by the Association of Universities for Research in Astronomy, Inc., for NASA, under contract NAS 5-26555.

\section{REFERENCES}

Abbott, T. et al. 2005

Abel, T., Bryan, G. L., \& Norman, M. L. 2002, Science, 295, 93

Abel, T. \& Haehnelt, M. G. 1999, ApJ, 520, L13

Alvarez, M. A., Busha, M. T., Abel, T., \& Wechsler, R. H. 2008, Apj (submitted) 0812.3405

Babul, A. \& Rees, M. J. 1992, MNRAS, 255, 346

Barkana, R. \& Loeb, A. 2004, ApJ, 609, 474
Belokurov, V., Walker, M. G., Evans, N. W., Faria, D. C., Gilmore, G., Irwin, M. J., Koposov, S., Mateo, M., Olszewski, E., \& Zucker, D. B. 2008, ApJ, 686, L83 
Belokurov, V., Zucker, D. B., Evans, N. W., Kleyna, J. T., Koposov, S., Hodgkin, S. T., Irwin, M. J., Gilmore, G., Wilkinson, M. I., Fellhauer, M., Bramich, D. M., Hewett, P. C., Vidrih, S., De Jong, J. T. A., Smith, J. A., Rix, H.-W., Bell, E. F., Wyse, R. F. G., Newberg, H. J., Mayeur, P. A., Yanny, B., Rockosi, C. M., Gnedin, O. Y., Schneider, D. P., Beers, T. C., Barentine, J. C., Brewington, H., Brinkmann, J., Harvanek, M., Kleinman, S. J., Krzesinski, J., Long, D., Nitta, A., \& Snedden, S. A. 2007, ApJ, 654, 897

Belokurov, V., Zucker, D. B., Evans, N. W., Wilkinson, M. I., Irwin, M. J., Hodgkin, S., Bramich, D. M., Irwin, J. M., Gilmore, G., Willman, B., Vidrih, S., Newberg, H. J., Wyse, R. F. G., Fellhauer, M., Hewett, P. C., Cole, N., Bell, E. F., Beers, T. C., Rockosi, C. M., Yanny, B., Grebel, E. K., Schneider, D. P., Lupton, R., Barentine, J. C., Brewington, H., Brinkmann, J., Harvanek, M., Kleinman, S. J., Krzesinski, J., Long, D., Nitta, A., Smith, J. A., \& Snedden, S. A. 2006, ApJ, 647, L111

Benson, A. J., Frenk, C. S., Lacey, C. G., Baugh, C. M., \& Cole, S. 2002, MNRAS, 333, 177

Blanton, M. R., Geha, M., \& West, A. A. 2008, ApJ, 682, 861

Blanton, M. R., Lupton, R. H., Schlegel, D. J., Strauss, M. A., Brinkmann,

J., Fukugita, M., \& Loveday, J. 2005, ApJ, 631, 208

Bode, P., Ostriker, J. P., \& Turok, N. 2001, ApJ, 556, 93

Bruzual, G. \& Charlot, S. 2003, MNRAS, 344, 1000

Bullock, J. S., Kolatt, T. S., Sigad, Y., Somerville, R. S., Kravtsov, A. V., Klypin, A. A., Primack, J. R., \& Dekel, A. 2001, MNRAS, 321, 559

Bullock, J. S., Kravtsov, A. V., \& Weinberg, D. H. 2000, ApJ, 539, 517

Busha, M. T., Evrard, A. E., \& Adams, F. C. 2007, ApJ, 665, 1

Chabrier, G. 2003, PASP, 115, 763

Colín, P., Avila-Reese, V., \& Valenzuela, O. 2000, ApJ, 542, 622

Conroy, C. \& Wechsler, R. H. 2009, ApJ, 696, 620

Conroy, C., Wechsler, R. H., \& Kravtsov, A. V. 2006, ApJ, 647, 201

Crocce, M., Pueblas, S., \& Scoccimarro, R. 2006, MNRAS, 373, 369

Dekel, A. \& Woo, J. 2003, MNRAS, 344, 1131

Diemand, J., Kuhlen, M., \& Madau, P. 2007a, ApJ, 657, 262

-. 2007b, ApJ, 667, 859

Diemand, J., Kuhlen, M., Madau, P., Zemp, M., Moore, B., Potter, D., \& Stadel, J. 2008, Nature, 454, 735

Dijkstra, M., Haiman, Z., Rees, M. J., \& Weinberg, D. H. 2004, ApJ, 601, 666

Drory, N. \& Alvarez, M. 2008, ApJ, 680, 41

Gao, L., Springel, V., \& White, S. D. M. 2005, MNRAS, 363, L66

Gardner, J. P., Connolly, A., \& McBride, C. 2007, in Astronomical Society of the Pacific Conference Series, Vol. 376, Astronomical Data Analysis Software and Systems XVI, ed. R. A. Shaw, F. Hill, \& D. J. Bell, 69-+ Geha, M. et al. 2008

Gnedin, N. Y. 2000, ApJ, 542, 535

Hahn, O., Porciani, C., Dekel, A., \& Carollo, C. M. 2008, ArXiv e-prints Haiman, Z. \& Bryan, G. L. 2006, ApJ, 650, 7

Iliev, I. T., Mellema, G., Pen, U.-L., Merz, H., Shapiro, P. R., \& Alvarez, M. A. 2006, MNRAS, 369, 1625

Iliev, I. T., Shapiro, P. R., \& Raga, A. C. 2005, MNRAS, 361, 405

Irwin, M. J., Belokurov, V., Evans, N. W., Ryan-Weber, E. V., de Jong, J. T. A., Koposov, S., Zucker, D. B., Hodgkin, S. T., Gilmore, G., Prema, P., Hebb, L., Begum, A., Fellhauer, M., Hewett, P. C., Kennicutt, Jr., R. C., Wilkinson, M. I., Bramich, D. M., Vidrih, S., Rix, H.-W., Beers, T. C., Barentine, J. C., Brewington, H., Harvanek, M., Krzesinski, J., Long, D., Nitta, A., \& Snedden, S. A. 2007, ApJ, 656, L13

Ishiyama, T., Fukushige, T., \& Makino, J. 2008, ArXiv e-prints Ivezic, Z. et al. 2008

Juneau, S., Glazebrook, K., Crampton, D., McCarthy, P. J., Savaglio, S., Abraham, R., Carlberg, R. G., Chen, H.-W., Le Borgne, D., Marzke, R. O., Roth, K., Jørgensen, I., Hook, I., \& Murowinski, R. 2005, ApJ, 619, L135

Kaiser, N., Aussel, H., Burke, B. E., Boesgaard, H., Chambers, K., Chun, M. R., Heasley, J. N., Hodapp, K.-W., Hunt, B., Jedicke, R., Jewitt, D., Kudritzki, R., Luppino, G. A., Maberry, M., Magnier, E., Monet, D. G., Onaka, P. M., Pickles, A. J., Rhoads, P. H. H., Simon, T., Szalay, A., Szapudi, I., Tholen, D. J., Tonry, J. L., Waterson, M., \& Wick, J. 2002, in Society of Photo-Optical Instrumentation Engineers (SPIE) Conference Series, Vol. 4836, Society of Photo-Optical Instrumentation Engineers (SPIE) Conference Series, ed. J. A. Tyson \& S. Wolff, 154-164

Kauffmann, G., White, S. D. M., \& Guiderdoni, B. 1993, Mon. Not. Roy. Astron. Soc., 264, 201

Keller, S. C. et al. 2007

Kepner, J., Tripp, T. M., Abel, T., \& Spergel, D. 1999, AJ, 117, 2063

Klypin, A., Kravtsov, A. V., Valenzuela, O., \& Prada, F. 1999, ApJ, 522, 82

Koch, A., McWilliam, A., Grebel, E. K., Zucker, D. B., \& Belokurov, V. 2008, ApJ, 688, L13
Komatsu, E., Dunkley, J., Nolta, M. R., Bennett, C. L., Gold, B., Hinshaw, G., Jarosik, N., Larson, D., Limon, M., Page, L., Spergel, D. N., Halpern, M., Hill, R. S., Kogut, A., Meyer, S. S., Tucker, G. S., Weiland, J. L., Wollack, E., \& Wright, E. L. 2008, ArXiv e-prints

Koposov, S., Belokurov, V., Evans, N. W., Hewett, P. C., Irwin, M. J., Gilmore, G., Zucker, D. B., Rix, H.-W., Fellhauer, M., Bell, E. F., \& Glushkova, E. V. 2008, ApJ, 686, 279

Koposov, S. E. et al. 2009, ArXiv e-prints

Kravtsov, A. V., Berlind, A. A., Wechsler, R. H., Klypin, A. A., Gottlöber, S., Allgood, B., \& Primack, J. R. 2004a, ApJ, 609, 35

Kravtsov, A. V., Gnedin, O. Y., \& Klypin, A. A. 2004b, ApJ, 609, 482

Li, Y.-S., Helmi, A., De Lucia, G., \& Stoehr, F. 2008, ArXiv e-prints

Lidz, A., McQuinn, M., Zaldarriaga, M., Hernquist, L., \& Dutta, S. 2007, ApJ, 670, 39

Maccio', A. V., Kang, X., Fontanot, F., Somerville, R. S., Koposov, S. E., \& Monaco, P. 2009, ArXiv e-prints

Maccio', A. V., Kang, X., \& Moore, B. 2008, ArXiv e-prints

Madau, P., Kuhlen, M., Diemand, J., Moore, B., Zemp, M., Potter, D., \& Stadel, J. 2008, ArXiv e-prints

Martin, N. F., de Jong, J. T. A., \& Rix, H.-W. 2008, ApJ, 684, 1075

Martin, N. F., Ibata, R. A., Chapman, S. C., Irwin, M., \& Lewis, G. F. 2007,

Mon. Not. Roy. Astron. Soc., 380, 281

Mateo, M., Olszewski, E. W., \& Walker, M. G. 2008, ApJ, 675, 201

Mateo, M. L. 1998, ARA\&A, 36, 435

Moore, B., Ghigna, S., Governato, F., Lake, G., Quinn, T., Stadel, J., \& Tozzi, P. 1999, ApJ, 524, L19

Noeske, K. G., Faber, S. M., Weiner, B. J., Koo, D. C., Primack, J. R., Dekel, A., Papovich, C., Conselice, C. J., Le Floc'h, E., Rieke, G. H., Coil, A. L., Lotz, J. M., Somerville, R. S., \& Bundy, K. 2007, ApJ, 660, L47

Orban, C., Gnedin, O. Y., Weisz, D. R., Skillman, E. D., Dolphin, A. E., \& Holtzman, J. A. 2008, ApJ, 686, 1030

Shaviv, N. J. \& Dekel, A. 2003, ArXiv Astrophysics e-prints

Simon, J. D. \& Geha, M. 2007, ApJ, 670, 313

Smith, J. A., Tucker, D. L., Allam, S. S., \& Jorgensen, A. M. 2002, in Bulletin of the American Astronomical Society, Vol. 34, Bulletin of the American Astronomical Society, 1272-+

Sokasian, A., Abel, T., \& Hernquist, L. 2002, MNRAS, 332, 601

Sokasian, A., Abel, T., Hernquist, L., \& Springel, V. 2003, MNRAS, 344, 607

Somerville, R. S. 2002, ApJ, 572, L23

Spergel, D. N., Bean, R., Doré, O., Nolta, M. R., Bennett, C. L., Dunkley, J., Hinshaw, G., Jarosik, N., Komatsu, E., Page, L., Peiris, H. V., Verde, L., Halpern, M., Hill, R. S., Kogut, A., Limon, M., Meyer, S. S., Odegard, N., Tucker, G. S., Weiland, J. L., Wollack, E., \& Wright, E. L. 2006, ArXiv Astrophysics e-prints

Springel, V. 2005, MNRAS, 364, 1105

Springel, V., Wang, J., Vogelsberger, M., Ludlow, A., Jenkins, A., Helmi, A., Navarro, J. F., Frenk, C. S., \& White, S. D. M. 2008, ArXiv e-prints Stadel, J., Potter, D., Moore, B., Diemand, J., Madau, P., Zemp, M., Kuhlen, M., \& Quilis, V. 2008, ArXiv e-prints

Strigari, L. E., Bullock, J. S., Kaplinghat, M., Diemand, J., Kuhlen, M., \& Madau, P. 2007a, ApJ, 669, 676

Strigari, L. E., Bullock, J. S., Kaplinghat, M., Simon, J. D., Geha, M., Willman, B., \& Walker, M. G. 2008, Nature, 454, 1096

Strigari, L. E., Koushiappas, S. M., Bullock, J. S., \& Kaplinghat, M. 2007b, Phys. Rev. D, 75, 083526

Taylor, J. E. \& Babul, A. 2004, MNRAS, 348, 811

Thoul, A. A. \& Weinberg, D. H. 1996, ApJ, 465, 608

Tollerud, E. J., Bullock, J. S., Strigari, L. E., \& Willman, B. 2008, ApJ, 688, 277

Tolstoy, E., Hill, V., \& Tosi, M. 2009, ArXiv e-prints

Walker, M. G., Mateo, M., Olszewski, E. W., Gnedin, O. Y., Wang, X., Sen, B., \& Woodroofe, M. 2007, ApJ, 667, L53

Walsh, S. M., Jerjen, H., \& Willman, B. 2007, ApJ, 662, L83

Walsh, S. M., Willman, B., \& Jerjen, H. 2009, AJ, 137, 450

Wechsler, R. H., Zentner, A. R., Bullock, J. S., Kravtsov, A. V., \& Allgood, B. 2006, ApJ, 652, 71

Weinmann, S. M., Macciò, A. V., Iliev, I. T., Mellema, G., \& Moore, B. 2007, MNRAS, 381, 367

Willman, B., Blanton, M. R., West, A. A., Dalcanton, J. J., Hogg, D. W., Schneider, D. P., Wherry, N., Yanny, B., \& Brinkmann, J. 2005a, AJ, 129, 2692

Willman, B., Dalcanton, J. J., Martinez-Delgado, D., West, A. A., Blanton, M. R., Hogg, D. W., Barentine, J. C., Brewington, H. J., Harvanek, M., Kleinman, S. J., Krzesinski, J., Long, D., Neilsen, Jr., E. H., Nitta, A., \& Snedden, S. A. 2005b, ApJ, 626, L85 
Willman, B., Governato, F., Dalcanton, J. J., Reed, D., \& Quinn, T. R. 2004, Mon. Not. Roy. Astron. Soc., 353, 639

Wise, J. H. \& Abel, T. 2008, ApJ, 685, 40

Zentner, A. R., Berlind, A. A., Bullock, J. S., Kravtsov, A. V., \& Wechsler, R. H. 2005, ApJ, 624, 505

Zentner, A. R. \& Bullock, J. S. 2003, ApJ, 598, 49

Zheng, X. Z., Bell, E. F., Papovich, C., Wolf, C., Meisenheimer, K., Rix, H.-W., Rieke, G. H., \& Somerville, R. 2007, ApJ, 661, L41

Zucker, D. B., Belokurov, V., Evans, N. W., Kleyna, J. T., Irwin, M. J.,

Wilkinson, M. I., Fellhauer, M., Bramich, D. M., Gilmore, G., Newberg, H. J., Yanny, B., Smith, J. A., Hewett, P. C., Bell, E. F., Rix, H.-W.,

Gnedin, O. Y., Vidrih, S., Wyse, R. F. G., Willman, B., Grebel, E. K.,

Schneider, D. P., Beers, T. C., Kniazev, A. Y., Barentine, J. C.,

Brewington, H., Brinkmann, J., Harvanek, M., Kleinman, S. J.,

Krzesinski, J., Long, D., Nitta, A., \& Snedden, S. A. 2006a, ApJ, 650, L41
Zucker, D. B., Belokurov, V., Evans, N. W., Wilkinson, M. I., Irwin, M. J., Sivarani, T., Hodgkin, S., Bramich, D. M., Irwin, J. M., Gilmore, G., Willman, B., Vidrih, S., Fellhauer, M., Hewett, P. C., Beers, T. C., Bell, E. F., Grebel, E. K., Schneider, D. P., Newberg, H. J., Wyse, R. F. G., Rockosi, C. M., Yanny, B., Lupton, R., Smith, J. A., Barentine, J. C., Brewington, H., Brinkmann, J., Harvanek, M., Kleinman, S. J.,

Krzesinski, J., Long, D., Nitta, A., \& Snedden, S. A. 2006b, ApJ, 643, L103 